\title{
Spinal Muscular Atrophy with Respiratory Distress Type 1: A Novel Variant of IGHMBP2 Gene
}

\author{
Muhammad Saeed, Walid Fawzy, Saeed Al-Tala, Tayseer Adel Magid and Hala Ahmed \\ Department of Pediatric Medicine, King Fahad Armed Forces Hospital, Khamis Mushait, Saudi Arabia
}

\begin{abstract}
Spinal muscular atrophy (SMA) with respiratory distress type 1 (SMARD1) is an exceptionally rare type of SMA. It results from disintegration of alpha motor neurons of the spinal cord. Clinically, children affected with this disorder present between the age of six weeks to six months with respiratory distress and hypotonia. Most of the children die before the age of 13 months. Here, we report a new variant in a female infant with SMARD1 having a novel IGHMBP2 gene mutation. Despite supportive treatment, she died at the age of 5 months in hospital. To the best of our knowledge, the variant has not been described in the literature so far.
\end{abstract}

Key Words: Spinal muscular atrophy with respiratory distress type-1 (SMARD1), Hypotonia, respiratory distress, infants

How to cite this article: Saeed M, Fawzy W, Tala SA, Magid TA, Ahmed H. Spinal Muscular Atrophy with Respiratory Distress

Type 1: A Novel Variant of IGHMBP2 Gene. J Coll Physicians Surg Pak 2021; 31(12):1494-1496.

\section{INTRODUCTION}

Spinal muscular atrophy (SMA) with respiratory distress type-1 (SMARD1) is an exceptionally rare disorder of spinal cord. It is also known as SMA distal type or distal hereditary motor neuropathy, type-5. SMARD1 is caused by autosomal recessive mutations in the gene encoding immunoglobulin $\mathrm{m}$-binding protein 2 (IGHMBP2, OMIM *600502) ${ }^{1,2}$ A cluster analysis of clinical symptoms showed that the probability of finding mutations in IGHMBP2 in patients with respiratory distress and suspected SMARD1 can be predicted by the following observations: manifestation age of respiratory distress between six weeks and six months, presence of diaphragmatic paralysis, distal muscular weakness, and intrauterine growth retardation. Most frequent clinical presentation is respiratory distress, which is progressive and may lead to the paralysis of diaphragmatic muscles. ${ }^{3}$ Progressive respiratory muscle weakness in these patients may end up in mechanical ventilation dependence. ${ }^{4}$

A variety of other symptoms and physical findings can occur in patients with SMARD1.

Herein, we describe a three-month female infant born to healthy, consanguineous parents with a unique and novel IGHMBP2 gene mutation, who developed progressive respiratory difficultly since the age of one month. To the best of our knowledge, the varianthas not been described in the literature so far.

Correspondence to: Dr. Muhammad Saeed, Department of Pediatric Medicine, King Fahad Armed Forces Hospital, Khamis Mushait, Saudi Arabia

E-mail: muhammad964@hotmail.com

Received: December 04, 2019; Revised: March 11, 2020;

Accepted: April 07, 2020

DOI: https://doi.org/10.29271/jcpsp.2021.12.1494

\section{CASE REPORT}

This is a case of a Saudi girl born at term by cesarean section due to a previous caesarean section with birth weight of $1.9 \mathrm{~kg}$. She was the product of a young first cousin Saudi couple with remarkable family history of male death at the age of six months with similar problems, but without any final diagnosis. Mother's antenatal scan showed normal liquor volume, and no congenital malformations were detected in the fetus but showed intrauterine growth retardation (IUGR) on repeated abdominal scan. She was thriving well until the age of two months, when the parents noted that she had feeding difficulties with frequent aspiration, so that by the age of 3 months, the mother had to use a thickening formula. Although the patient had a weak cry from early on, this also worsened with the deterioration of motor activity. At the age of three months, she was admitted to our hospital with respiratory distress and feeding difficulties.

She was treated with positive pressure ventilation; but due to gradual deterioration of respiratory functions, she was intubated and connected to mechanical ventilation (Figure 1).

The patient's weight, height, and head circumference were all at third percentile. On physical examination, she was active, alert, fixing and following visual stimuli, and interacting with her surroundings. She also showed minimal tongue fasciculation. There was generalised decrease in muscle bulk, more prominent in the distal muscle groups associated with marked hypotonia, more evident in the limbs.

Flexion contractures were noted over ankle and knee joints (Figure 2). Power in all limb muscles was of grade three with absent deep tendon reflexes. Her all accessible cranial nerves were intact with clinically normal vision and hearing.

The patient had a normal thoracic cage shape and her cardiovascular examination was normal, except for unexplained episodes of tachycardia. 


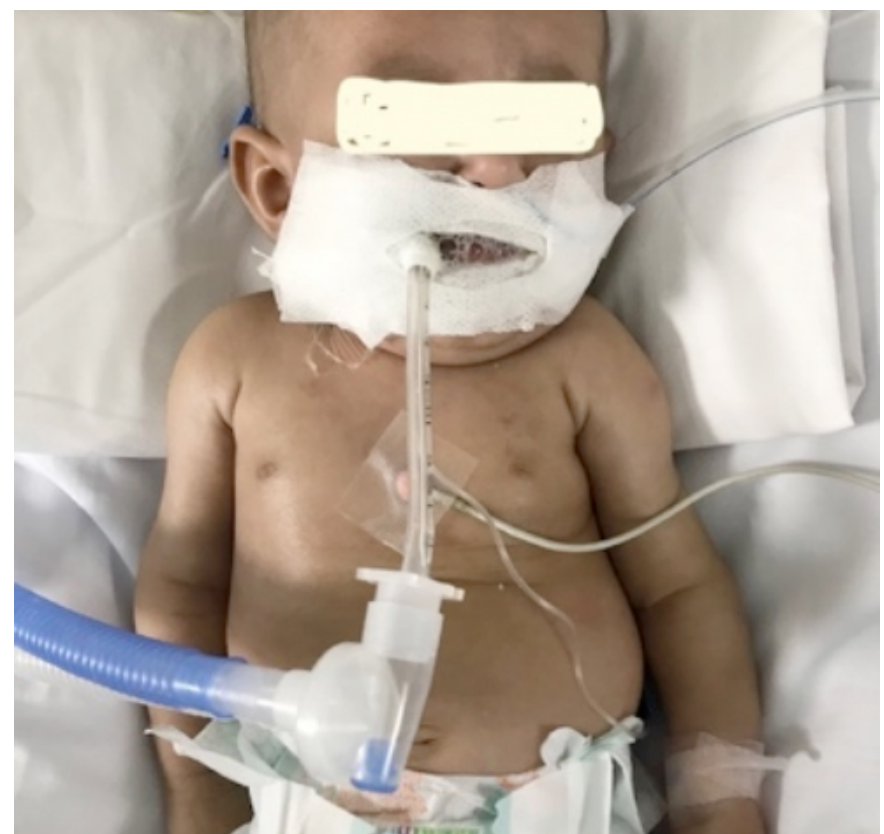

Figure 1. Mechanical ventilation dependency of the infant.

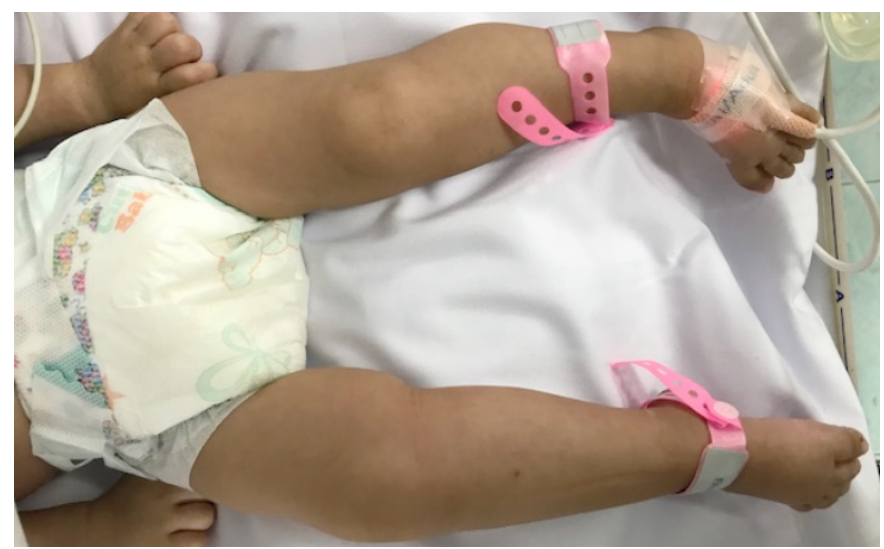

Figure 2. Distal lower limb muscle wasting with contractures.

An initial workup, including lactate levels, plasma creatine phosphokinase (CPK), serum ammonia, serum and cerebrospinal fluid (CSF) lactate, urine organic acids, long chain fatty acids, acylcarnitine, respiratory chain enzymes, and urine organic acids, were all within normal range.

Apart from the pneumonic changes, her chest radiograph showed eventration of the right hemi-diaphragm.

Electrocardiogram, echocardiography and abdominal ultrasound examinations were normal. The initial working diagnosis was infantile SMA; however, genetic study showed no SMN gene mutations.

Her genetic study, by whole exome sequencing, identified the homozygous 1-bpduplicationvariantc. 797 dupGp.(His267Thrfs*47) (chr11:68682373; hg19) in the IGHMBP2 gene. No allele frequencies in the general population have been documented and this is the first time it was detected with the same variant.

Patient died at the age of 5 months in hospital. Her parents were also evaluated by segregation analysis and they were confirmed carriers of IGHMBP2 gene mutation.

\section{DISCUSSION}

Mutations in the IGHMBP2 gene encoding the immunoglobulin $\mathrm{m}$-binding protein 2 on chromosome 11q13.3 were identified in 2001. Since then, more than 60 cases with distal SMA type 1 (SMA1), have been documentd in literature. Both SMA type 1 and SMARD1 diseases cause degeneration of the anterior horn cells in the spinal cord resulting in progressive weakness and wasting of muscle, but SMARD1 is distinct genetically and clinically from SMA1 (Werdnig-Hoffman disease). The prevalence of SMARD1 is unknown, but Rudnik-Scho"neborn et al. estimated that diaphragmatic paralysis could affect largely $1 \%$ of patients with early-onset SMA. There is early respiratory distress and failure due to paralysis or eventration of diaphragm in SMARD1, which manifests before the weakness of distal muscles. ${ }^{5}$

Grohmann et al. found mutations of IGHMBP2 in patients who presented with hypotonia, progressive respiratory distress and they proposed that these mutations are the cause of SMARD1. They discovered 26 novel IGHMBP2 mutations.2,3 Maystadt et al. identified nine novel IGHMBP2 mutations. ${ }^{4}$

In 2003, Pitt et al. defined the clinical characteristics of SMARD1 based on clinical symptoms which include IUGR, progressive respiratory distress due to diaphragm paralysis at the age of one to six months, and progressive muscular weakness, predominantly involving distal lower limbs. ${ }^{6}$ In our case, the parents were first-degree cousins and the gene mutation detected was a homozygous variant c. 797 dupG p.(His267Thrfs*47) (chr11:68682373; hg19) in exon 7 of the IGHMBP2 gene. Many cases of SMARD1 born to consanguineous parents of Middle Eastern background have been reported in the literature. $^{7}$

In Saudi Arabia, the overall rate of consanguineous marriage is reported to be $57.7 \%$, with regional variation from as low as $34 \%$ to an astounding high of $80.6 \%$. The most prominent presenting symptom of spinal SMARD1 is a rapidly progressive and lifethreatening severe respiratory distress that results from diaphragmatic paralysis. Respiratory failure developed at the age of three months in our case, which is in accordance with previous reports demonstrating that the paralysis of the diaphragm does not occur congenitally but most often in the first months of life and results irreversibly in the necessity for mechanical ventilation. ${ }^{8}$ In a study on constellations that render the presence of an IGHMBP2 gene mutation likely, the single most important criterion for the presence of an IGHMBP2 gene mutation was a congenital manifestation of respiratory failure. ${ }^{9}$ Guenther et al. reported 141 cases with SMARD1 constellation of symptoms that predicts the presence of IGHMBP2 gene mutations, especially in case of a patient born to consanguineous parents. ${ }^{10}$

The autonomic nervous system dysfunction, reported earlier in many cases of SMARD1, was observed in our case in the form of 
bouts of changes in heart rate. In countries with high consanguineous marriage and similar case scenario, where it might be difficult to perform a costly and rarely needed test such as IGHMBP2 mutation sequencing, it will be of help to first exclude SMN1 exon 7 deletion SMA and to consider other supportive tests. If these test results are in favour of SMARD1 diagnosis, then IGHMBP2 mutation screening can be ordered. . $^{10,11}$

In conclusion, we believe that the syndrome is underdiagnosed and the clinicians practising in areas with high consanguineous marriages, need to be alerted to its possibility. A clinical picture of infantile hypotonia with a predominant involvement of distal limb muscles, demonstrated to be of neurogenic origin, should raise the suspicion of SMARD1, especially in the case of a patient born to consanguineous parents.

\section{PATIENT'S CONSENT:}

Informed consents were obtained from the patient's parents to publish this case report.

\section{CONFLICT OF INTEREST:}

The authors declared no potential conflicts of interests with respect to the authorship and/or publication of this article

\section{AUTHORS' CONTRIBUTION:}

MS: Design of work, interpretation, collection and analysis, and review of literature.

WF: Collected and provided updated literature, and reviewed the report.

SAT, TAM, HA: Reviewing the literature, drafting the work, and revising it critically for important for intellectual content.

\section{REFERENCES}

1. Bertini E, Gadisseux JL, Palmieri G, Ricci E, Di Capua M, Ferriere G, et al. Distal infantile spinal muscular atrophy associated with paralysis of the diaphragm: A variant of infantile spinal muscular atrophy. Am J Med Genet 1989; 33(3):328-35. doi: 10.1002/ajmg.1320330309.

2. Grohmann K, Schuelke M, Diers A, Hoffmann K, Lucke B, Adams $C$, et al. Mutations in the gene encoding immunoglobulin mu-binding protein 2 causing spinal muscular atrophy with respiratory distress type-1. Nat Genet 2001; 29(1):75-7. doi: 10.1038/ng703.
3. Grohmann K, Varon R, Stolz P, Schuelke M, Janetzki C, Bertini $\mathrm{E}$, et al. Infantile spinal muscular atrophy with respiratory distress type-1 (SMARD1). Ann Neurol 2003; 54(6):719-24. doi: 10.1002/ana.10755.

4. Maystadt I, Zarhrate M, Landrieu P, Boespflug-Tanguy O, Sukno $S$, Collignon $P$, et al. Allelic heterogeneity of SMARD1 at the IGHMBP2 locus. Hum Mut 2004; 23(5): 525-6. doi: 10.1002/humu.9241.

5. Rudnik-Scho"neborn S, Stolz P, Varon R, Grohmann K, Schächtele $M$, Ketelsen U-P, et al. Long-term observations of patients with infantile spinal muscular atrophy with respiratory distress type 1 (SMARD1). Neuropediatrics 2004; 35(3):174-82. doi: 10.1055/s- 2004-820994.

6. Pitt M, Houlden H, Jacobs J, Mok Q, Harding B, Reilly M, et al. Severe infantile neuropathy with diaphragmatic weakness and its relationship to SMARD1. Brain 2003; 126(Pt 12):2682-92. doi: 10.1093/brain/awg278.

7. Tachi N, Kikuchi S, Kozuka N, Nogami A. A new mutation of IGHMBP2 gene in spinal muscular atrophy with respiratory distress type 1. Pediatr Neurol 2005; 32(4):288-90. doi: 10.1016/j.pediatrneurol.2004.11.003.

8. Wong VC, Chung BH, Li S, Goh W, Lee SL. Mutation of gene in spinal muscular atrophy respiratory distress type-1. Pediatr Neurol 2006; 34(6):474-7. doi.org/10. 1016/j.pediatrneurol.2005.10.022

9. Joseph S, Robb SA, Mohammed S, Lillis S, Simonds A, Manzur AY, et al. Interfamilial phenotypic heterogeneity in SMARD1. Neuromuscul Disord 2009; 19(3):193-5. doi: 10.1016/j. nmd.2008.11.013.

10. Guenther UP, Varon R, Schlicke M, Dutrannoy V, Volk A, Hübner $\mathrm{C}$, et al. Clinical and mutational profile in spinal muscular atrophy with respiratory distress (SMARD1): Defining novel phenotypes through hierarchical cluster analysis. Hum Mutat 2007; 28(8):808-15. doi: 10.1002 /humu.20525.

11. Guenther UP, Handoko L, Varon R, Stephani U, Tsao CY, Mendell JR, et al. Clinical variability in distal spinal muscular atrophy type-1 (DSMA1): Determination of steady-state IGHMBP2 protein levels in five patients with infantile and juvenile disease. J Mol Med 2009; 87(1): 31-41. doi: 10.1007/s00109-008-0402-7. 